\title{
Situación del acceso abierto en las universidades. Caso y modelo de análisis Universidad de Antioquia
}

Situation of open access in universities. Case and model of analysis at University of Antioquia

\section{Alejandro Uribe-Tirado}

Escuela Interamericana de Bibliotecología,

Universidad de Antioquia, Medellin, Colombia

alejandro.uribe2@udea.edu.co

\section{Jaider Ochoa-Gutiérrez}

Escuela Interamericana de Bibliotecología,

Universidad de Antioquia, Medellin, Colombia

jaider.ochoa@udea.edu.co

\section{César Pallares}

Universidad de Antioquia, Colombia

cesar.pallares@udea.edu.co

\section{Diego Restrepo-Quintero}

Instituto de Física, Universidad de Antioquia, Medellín, Colombia

restrepo@udea.edu.co

\section{Gabriel Vélez-Cuartas}

Facultad de Ciencias Sociales, Universidad de Antioquia, Medellin, Colombia

gjaime.velez@udea.edu.co

\section{Jorge Robledo-Velásquez}

Facultad de Minas, Universidad Nacional de Colombia, Medellín, Colombia

jrobledov@unal.edu.co

\section{Huber Gómez-Molina}

Sistema de Bibliotecas, Universidad de Antioquia, Medellin, Colombia

huber.gomez@udea.edu.co

\section{Maria Coral Correa-Tabares}

Sede de Investigación Universitaria, Universidad de Antioquia, Medellín, Colombia

coral.correa@udea.edu.co

\section{Julián Calle-Mosquera}

Instituto de Fisica, Universidad de Antioquia, Medellin, Colombia

julian.callem@udea.edu.co

\section{Ana María Osorio-López}

Facultad de Ciencias Sociales, Universidad de Antioquia, Medellin, Colombia

amaria.osorio@udea.edu.co 


\section{ReSUMEN:}

Desde los noventa, el movimiento de acceso abierto ha impulsado la democratización y el acceso global a la investigación partiendo de prácticas y herramientas que buscan romper las barreras al acceso y uso de las publicaciones científicas. Esto ha generado modificaciones en las formas de comunicación científica de los investigadores, por lo que las instituciones requieren estrategias de medición que les permitan dimensionar estos cambios. Por ello, el objetivo de este artículo es proponer una metodología para medir y caracterizar las prácticas de acceso abierto en las universidades latinoamericanas, tomando como caso de estudio la Universidad de Antioquia. Para ello, se diseñó una estrategia de recopilación automática de datos bibliográficos, de tres fuentes (Web of Science, SciELO y Scopus), y se calcularon indicadores de acceso abierto, para 7.990 artículos con DOI de la Institución. Se identificó que la producción abierta de la Universidad se ha incrementado en los últimos años, especialmente en el caso de la ruta dorada, con incidencia en el pago de APC realizado por los documentos académicos.

Palabras clave: APC, Acceso abierto, DOI, Universidad de Antioquia, Publicación científica.

\section{Abstract:}

Since the 1990s, the open access movement has promoted democratization and global access to research based on practices and tools that seek to break down the barriers to access and use of scientific publications. This has led to changes in the forms of scientific communication of researchers, so institutions require measurement strategies that allow them to dimension these changes. Therefore, the objective of this article is to propose a methodology to measure and characterize open access practices in Latin American universities, taking the University of Antioquia as a case study. For this, an automatic bibliographic data collection strategy was designed from three sources (Web of Science, SciELO and Scopus), and open access indicators were calculated for 7,990 articles with DOI of the Institution. It was identified that the open production of the University has increased in recent years, especially in the case of the golden route, with an impact on the payment of APC made by academic documents.

KEYWORDS: APC, Open access, DOI, Universidad de Antioquia, Research publication.

\section{INTRODUCCIÓN ${ }^{1}$}

El funcionamiento tradicional de la comunicación científica decantó en que unas cuantas editoriales comerciales controlaran en 2013 un 53\% de las revistas académicas, lo que les ha permitido cobrar suscripciones excesivas para acceder a los artículos y, por tanto, ha limitado la posibilidad de democratizar el conocimiento y de hacerlo libremente accesible a la sociedad (Resnick y Belluz, 2019). Como respuesta, la comunidad académica ha impulsado desde los noventa el movimiento de acceso abierto con el fin de instaurar prácticas y herramientas que rompan con esas barreras y posibiliten, así, la distribución y el uso de las publicaciones. Este movimiento se ha reflejado en declaraciones internacionales como las de Budapest (2002), Bethesda (2003) y Berlín (2003), que han puesto de manifiesto la necesidad de abrir las publicaciones científicas y han dado la ruta para consolidar la filosofía del movimiento (Abadal, 2012).

A partir de esta filosofía, la comunidad académica ha decantado tres rutas para el acceso abierto como respuesta al acceso cerrado: ruta dorada, ruta verde y ruta híbrida. La ruta dorada corresponde a la publicación en revistas que permite el acceso sin restricciones al artículo en texto completo (Suber, 2008). La ruta verde promulga el depósito de las publicaciones en repositorios digitales (temáticos o institucionales) que son operados principalmente por universidades, sociedades científicas o centros de investigación (Harnad et al., 2008). La ruta hibrida define la publicación abierta de artículos en revistas cerradas, siempre y cuando los autores paguen un costo económico por la publicación y/o liberación, lo que se ha denominado Article Procesing Charge -APC- (Schiermeier y Rodriguez Mega, 2017; Laakso y Björk, 2013), y que pueden 
implicar nuevas formas en que las instituciones terminan financiando las ganancias de las grandes casas editoriales.

En el desarrollo de estas rutas ha sido posible construir varias iniciativas con el fin de facilitar la identificación de canales para publicar en abierto. Entre estos se encuentran: DOAJ (Directorio de Revistas en Acceso Abierto), que sirve de fuente de identificación de este tipo de revistas; OpenAire, que centraliza los repositorios; y OpenDOAR, que permite identificar las políticas de acceso abierto de repositorios alrededor del mundo. En el caso latinoamericano, se han impulsado iniciativas como SciELO y RedALyC que buscan indexar revistas regionales de la ruta dorada, con lo cual estas plataformas han apoyado la visibilidad, a la vez que han puesto de manifiesto la necesidad de promover la apertura de la producción y las múltiples posibilidades de sostenibilidad y consolidación de fuentes abiertas.

No obstante, esto ha creado también una situación problemática: en la medida que surgen distintas fuentes de información cada una genera y ofrece datos propios. Incluso, estas fuentes pueden incluir la misma información o ser complementarias entre sí, lo que genera presiones en las instituciones para elegir una de ellas y realizar sus estrategias de política a partir de éstas. Por otro lado, el acceso a información normalizada se ha convertido en una nueva oportunidad de negocios para las casas editoriales, quienes ofrecen a las instituciones servicios como InCites (Clarivate Analytics) o SciVal (Elsevier) que permiten la construcción de indicadores para hacer seguimiento de su desempeño.

Actualmente, todas las instituciones requieren información confiable para su toma de decisiones, sin embargo, no todas tienen la oportunidad de acceder a estos servicios. Tal es el caso de la Universidad de Antioquia (UdeA) una institución pública colombiana con altas capacidades de investigación ${ }^{2}$, en cuyo Plan de Desarrollo Institucional 2017-2026, apuesta por la ciencia abierta como uno de sus lineamientos estratégicos, por lo que requiere de información cuantitativa para medir si está cumpliendo con dicha estrategia.

Este trabajo pretende abordar las dos situaciones problemáticas mencionadas. Para ello, en primer lugar, presenta un diseño metodológico en el que se extrae información de varias fuentes, se depura y, luego, se genera una base de datos centralizada de producción científica institucional, identificando cuál está disponible en acceso abierto, por cuál de las rutas se está concretando y cuál es el valor del pago de APC que se está realizando. Cabe aclarar que la herramienta que se ha desarrollado está publicada en abierto, por lo que, cualquier institución puede acceder y utilizarla para sus intereses. En segundo lugar, presenta una descripción de la producción en acceso abierto de la UdeA en la que se analiza cómo ha variado en el tiempo, y con base en la cual, luego se esgrimen una serie de recomendaciones a la institución para que esta pueda tomar decisiones, en políticas y estrategias, con el fin de cumplir el objetivo de su Plan de Desarrollo.

\section{UNIVERSIDAD Y ACCESO ABIERTO}

La relación entre universidad y el acceso abierto ha estado siempre presente desde que el movimiento open access inició a mediados de los noventa. Ésta ha sido el resultado de las posturas por una ciencia más accesible para todos, realizando esfuerzos de la comunidad académica para cambiar el rumbo de la comunicación científica la cual ha estado marcada por tres hechos fundamentales: el abuso de los oligopolios editoriales (Luchilo, 2019) ${ }^{3}$, el potencial que ofrece Internet como un modo emergente de la comunicación científica -modo 2- (Briceño, 2013; Gibbons, 2000), y el marco general de la ciencia abierta donde el acceso abierto es uno de sus componentes claves e impulsores (Anglada y Abadal, 2018) ${ }^{4}$. Estos tres hechos y diferentes hitos ${ }^{5}$ han generado que estas últimas décadas sean un período con muchos movimientos, cambios y conflictos, sobre los que hay que ir adaptándose y respondiendo, asumiendo posturas y aprendiendo, en cuanto hacia dónde va la comunicación científica, y el papel y las decisiones que implica a las universidades 
como institución académica-científica, a los profesores-investigadores como autores y evaluadores, y a sus revistas-editoriales como difusores y divulgadores.

En particular, las universidades han tenido un papel fundamental en el desarrollo del acceso abierto, considerando la ruta dorada y verde. En la ruta dorada, las universidades han sido el origen de la mayoría de las revistas de acceso abierto sin cobro ${ }^{6} \mathrm{y}$, en la verde, han dado lugar a la mayor parte de repositorios institucionales ${ }^{7}$, e incluso, han estado involucradas en la creación de los repositorios temáticos si consideramos que éstos son el esfuerzo de comunidades académico-científicas que se vinculan ${ }^{8}$. Estos espacios (revistas y repositorios) son los que han ido posibilitado que el acceso al conocimiento científicoacadémico en los últimos años esté cada vez más disponible.

Desafortunadamente, en este proceso dialéctico y de aprendizajes, el espíritu original del acceso abierto, del conocimiento para todos, sin ningún tipo de costo con unas adecuadas disponibilidades legales o tecnológicas, se ha complicado puesto que las mismas cinco empresas que concentran el mercado de la comunicación científica -Reed/Elsevier, Wiley/Blackwell, Springer, y Taylor \& Francis- (Larivière, Haustein y Mongeon, 2015), tras varios años de "saboteo" a este movimiento abierto con mitos tales como: "si es en acceso abierto, es de baja calidad", entre otros (Tennant et al, 2019); han cambiado su modelo de negocio, creando y posicionando el de APC. Este cambio que desde el punto de vista del lector puede ser visto como "positivo", ya que ahora le es posible acceder libremente a lo que antes solo mediante suscripciones muy costosas a bases de datos o por la compra de la descarga de un artículo específico; es, sin embargo, desde el punto de vista de los autores y las instituciones "negativo", ya que resulta en un sobrecosto, que implica gastos o pocas posibilidades para la publicación al no contar con los recursos económicos necesarios para financiar los APCs.

En este marco es preciso recordar que una de las principales razones para la emergencia del acceso abierto fue la "crisis de las suscripciones" en la década de los noventa para las universidades, las cuales debían realizar grandes inversiones, para poder acceder a las publicaciones. Dicha situación en la actualidad para las instituciones no ha mejorado, puesto que se pasó de un cobro, a dos cobros: Suscripción a las bases de datos, que pagan las bibliotecas más Pagos por el $A P C$, que pagan los investigadores, grupos de investigación, instituciones $\mathrm{y} / \mathrm{o}$ financiadores ${ }^{9}$; lo cual ha llevado a la necesidad de que las universidades $-\mathrm{y}$ también los organismos de ciencia de cada país o de regiones- deban plantearse algunas acciones necesarias e interrelacionadas, en dos frentes:

1. Realizar mediciones propias que den cuenta de su realidad respecto al acceso abierto, tanto a nivel de la ruta dorada -revistas- como de la ruta verde -especialmente, su repositorio institucional-; su inversión en APC; su visibilidad e impacto, entre otras; y así, establecer estrategias institucionales a corto, mediano y largo plazo de comunicación científica.

2. Con información más precisa y cercana a cada contexto (región y/o institución), renegociar con esas empresas, para bajar costos de suscripción e incluir dentro del "paquete" también el APC; buscar nuevas alternativas; o romper contratos con dichas empresas ${ }^{10}$.

En este sentido este artículo --y la investigación que lo sustenta- tiene por objetivos desarrollar un modelo de captura y análisis de datos que permita a una universidad (para este estudio, la Universidad de Antioquia) identificar su situación respecto al acceso abierto de sus publicaciones, sus tipologías y costos, y la visibilidad e impacto de esa producción. De esta forma se busca responder ala primera acción propuesta (mediciones propias para una información más precisa) y hacer un aporte para que la segunda acción pueda mejorar su proceso e implementarse. En el caso específico de la Universidad de Antioquia, para que su Sistema de Bibliotecas, que ha sido uno de los líderes en los últimos meses para conformar un consorcio entre 60 universidades colombianas ${ }^{11}$, busque mejores precios con las bases de datos, y en un futuro cercano, cuente con información para la negociación de suscripciones, la reducción o eliminación de los costos APC. 
Antes de finalizar este apartado es necesario indicar que una parte de las transformaciones actuales que lleva la relación entre universidad y acceso abierto, es el reconocimiento que las universidades deben hacer, de la visibilidad e impacto de su producción científica (la vinculación que tiene con el entorno, dada a partir de su producción), una estrategia fundamental apoyada desde diferentes dependencias, pero integradora y holística, ya que hasta antes del 2010 era centrada solamente en indicadores bibliométricos. Sin embargo, ahora es necesario considerar nuevas opciones complementarias a lo bibliométrico como son las altmetrics, que permiten identificar la visibilidad e impacto no solo en el entorno científico -otros pares investigadoressino también en otros entornos - profesionales, medios de comunicación, políticos, ciudadanía, etc.-. Es decir, conocer la realidad del acceso abierto de una universidad, si se quiere hacerlo de manera integral y holística, implica tener claro, tanto el nivel de producción en relación al acceso abierto (sea en la ruta dorada o en la ruta verde), como los costos o no qué está implicando el acceso abierto considerando el APC (ruta hídrida), y conocer la visibilidad y el impacto de esa producción, y cómo el acceso abierto lo podría incrementar (Piwowar et al, 2018).

\section{REFERENTES DE MEDICIÓN DEL ACCESO ABIERTO}

Tras una revisión del tema respecto a cómo se ha medido el acceso abierto en diferentes contextos y niveles, se encontraron varios trabajos que resultan claves tanto para la metodología y el modelo propuesto como para comprender los datos específicos de la Universidad de Antioquia ${ }^{12}$.

Estos trabajos son referentes para este estudio en distintos niveles: diagnóstico universitario, de país y mundial, costos del acceso abierto bajo una ruta y propuesta de indicador del grado de acceso abierto. En el primer nivel, como diagnóstico universitario, se destaca el trabajo Koskinen et al. (2010), quienes presentan un estado de la situación del acceso abierto para el caso específico de la University of Helsinki (Finlandia). El mismo hace un análisis sobre la situación respecto al acceso abierto de toda su producción científica en los últimos años, las posibilidades de acceder a la producción científica abierta, del cómo se da la situación a nivel de las distintas facultades, etc. Esta investigación permite conocer a nivel de institución universitaria, qué tan abierta está siendo su producción e identificar las dinámicas, la apropiación y las posibilidades del acceso abierto, que resultan no ser semejantes en todas las facultades y disciplinas, como también otros trabajos lo han dado a conocer (López-Borrull, 2012; García Martín, 2015; González, Reverter-Masia y Jové-Deltell, 2017; entre otros); y muestran así, la necesidad de indagar los niveles de acceso abierto para las universidades como un todo, pero también a nivel de facultades.

En segundo lugar, ya a nivel de países, se debe mencionar el reciente trabajo de Hook, Calvert y Hahnel (2019), el cual presenta desde los datos que ofrece Dimensions, una visión de la situación en cuanto a revistas de diferentes países, respecto al acceso abierto, los tipos de acceso abierto, la colaboración, los resultados altmétricos, etc. Este trabajo es importante, ya que considerando la ruta dorada, sea desde revistas de la propia institución o de las revistas donde los investigadores publican, permite identificar la tendencia más hacia un modelo de publicación cerrado o de acceso abierto, es decir, ¿qué tipo de publicación es más usado por los investigadores?, ¿qué tendencia a nivel de citación conlleva el publicar en un modelo u otro?, y específicamente, cuando es de acceso abierto, ¿qué tipo de acceso abierto?, ¿qué ruta se estaría privilegiando? como ya lo han caracterizado algunos autores ${ }^{13}$, o qué cambios (ventajas y amenazas) se estarían dando al aparecer otras rutas, más allá del acceso por ruta dorada, como la hemos entendido y asumido mayoritariamente en América Latina (Debat y Babini, 2019). Asimismo, este trabajo permite identificar qué tanta producción está o no en abierto e identificar los tipos de abierto (rutas) y las implicaciones en citación o en costos (APC y otros), según la decisión de sus investigadores de dónde publicar, o los prerrequisitos de financiación o no, que tengan las mismas universidades o los organismos de ciencia y financiación de la investigación en cada país o regiones del mundo. 
En tercer lugar, pero ya desde un aspecto específico y crítico en estos momentos, como el APC -Modelo Plan S vs AmeliCA- (Debat y Babini, 2019), se destaca el trabajo de Morrison et al, que desde el 2015, llamaban la atención de esta situación, de esta ruta híbrida, al identificar tomando como base DOAJ, la situación de las revistas de acceso abierto frente al cobro o no de APC y sus rangos de $\operatorname{cobros}^{14}$. Estos trabajos, respecto al APC son claves actualmente si se quiere profundizar en la medición del acceso abierto, ya que no es solo qué tanta producción está finalmente en abierto para el usuario-lector, sino, si para acceder a la misma, hubo algún costo indirecto y/o si se asumió un modelo mixto; considerando la importancia de identificar si el aspecto original de gratuidad para todos, no se está dando para los agentes que generan la producción científica o para quienes lo financian, y por ello, los altos pagos institucionales por APC. Conocer hoy este costo en una universidad o para un financiador es un aspecto fundamental teniendo en cuenta la crítica por los dobles, triples y hasta cuádruples pagos que los oligopolios de la comunicación científica están imponiendo (Luchilo, 2019), o que las mismas universidades e investigadores se han ido imponiendo, o considerando las posturas poco críticas de nuestros organismos de ciencia y los otros agentes de la comunicación científica actual (Toledo, 2014).

Finalmente, para esta revisión y acercamiento a qué medir y cómo en acceso abierto, se destaca la propuesta de Nichols y Twidale (2017), quienes proponen el indicador Openness Index para identificar el grado de acceso abierto, el cual puede aplicarse a nivel de investigador, de facultad, de universidad, de país o comparativo de regiones, etc.; siempre y cuando se tenga la información para identificar las variables de dicho indicador (Figura 1).

FIGURA 1

Indicador: Openness Index

\section{Openness Index}

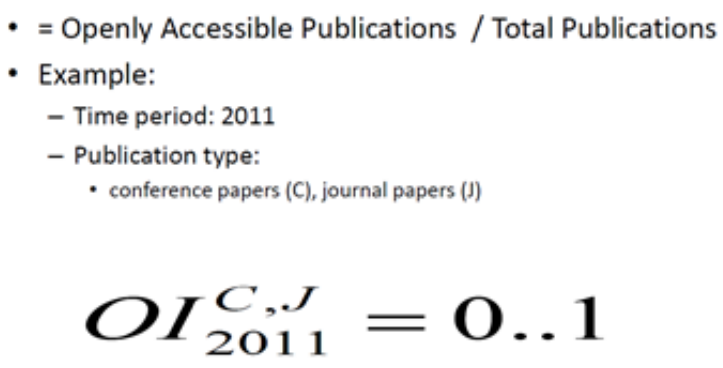

Fuente: Nichols y Twidale, 2017.

Los trabajos que se han reseñado constituyen los referentes teórico-conceptuales y metodológicos que más aportan a este trabajo, que buscó, como se ha mencionado, presentar una metodología de cómo es posible responder a la pregunta: ¿Cuál es la situación de acceso abierto de la universidad...? y se aplicó al caso de la Universidad de Antioquia (Medellín-Colombia). Cabe aclarar que, si bien se tomaron como base aportes de estos cuatro trabajos, en el modelo que se presenta se realizaron nuevas propuestas, especialmente, interrelacionando más datos, perspectivas, fuentes, etc.; lo que lo hace más integral y holístico, y replicable para otras universidades. 


\section{Proceso metodológico y tamaño de la Población}

Para realizar una mirada integral de la situación de acceso abierto, en este caso para la Universidad de Antioquia (UdeA), definiéndolo como modelo para que otras universidades pueden seguirlo para identificar su propia situación a partir de diferentes datos, se determinaron seis pasos(Figura 2):

FIGURA 2

Estrategia metodológica: Pasos y precisiones de datos para el caso de la UdeA

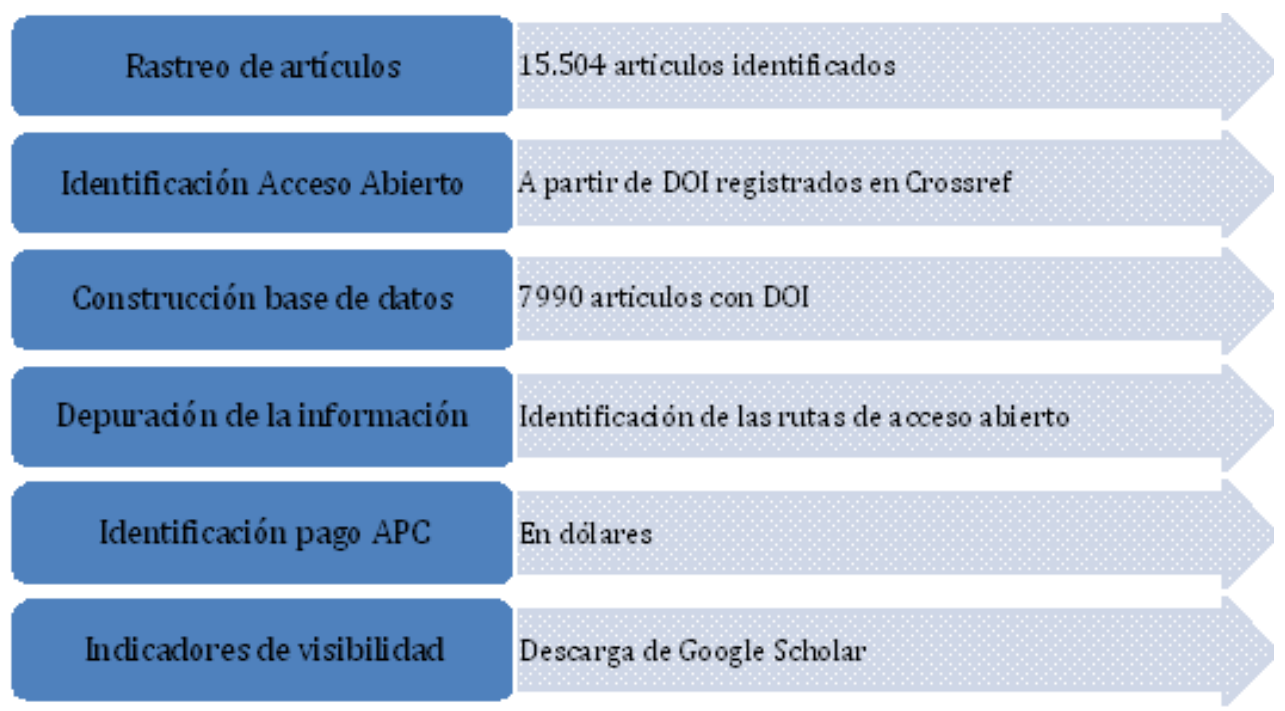

Fuente: elaboración propia.

A continuación, se explican cada uno de los pasos en relación con el caso estudiado:

\section{Rastreo de artículos}

Se toman las variantes de la firma institucional de los autores de la Universidad y se realiza una búsqueda de su producción en las bases de datos Web of Science (WOS), SciELO (para aquellas revistas que están en el SciELO Citation Index) y Scopus ${ }^{15}$. Estas bases fueron cruzadas entre sí a partir del DOI (Digital Object Identifier) de los artículos, con lo que fue posible identificar la producción científica de la Universidad, correspondiente a 15.504 artículos diferentes (hasta 2017). Para conseguir esto se construyó un algoritmo de extracción de información usando Jupyter ${ }^{16}$. Asimismo, corresponde aclarar que estas bases fueron seleccionadas por razones de cobertura, puesto que incluyen producción de corriente principal, es decir, que hace parte de los canales de comunicación científica de las ciencias duras en países anglosajones, pero también de nuestros contextos, considerando los artículos más enfocados a tener pertinencia regional en Latinoamérica.

Como se ha reiterado, el seguimiento a la situación de acceso abierto es una necesidad para conocer la visibilidad y el impacto que la producción científica que se genera desde la institución está teniendo. Tal como lo indica el documento REBIUN (2019), existen al menos dos formas de hacerlo a partir de los CRIS o de las API. Para este caso, por el momento, se debió recurrir a la segunda forma, pero es claro que esta metodología debe ser un proceso continuo desde los CRIS, que en el caso de la Universidad de Antioquia estará apoyado por el COLAV (Colaboratorio de Ciencias Sociales Computacionales).

2. Identificación de producción UdeA 
A partir de esta producción única, el siguiente paso consistió en identificar los artículos que fueron publicados en acceso abierto. Esto implicó un trabajo de ensayo y error con distintas herramientas y aplicaciones existentes en la literatura. Finalmente, se decidió que el método más eficiente era hacerlo a través del DOI de los artículos ${ }^{17}$. Para lograr esto, la herramienta que se utilizó fue oaDOI.org ${ }^{18}$, puesto que incluye registros para 88 millones de DOIs en Crossref.

\section{Construcción de base de datos}

Combinando los pasos 1 y 2, se identificó la cantidad de artículos de la Universidad que cuentan con DOI, llegando a un tamaño de 7.990 artículos para la institución, lo que significa un 51,5\% del total de artículos únicos alojados en las bases de datos. Sobre esta población se construyó una variable dicotómica que podía tomar dos valores: falso o verdadero, dependiendo si estos estaban en alguna de las rutas del acceso abierto o no. De igual manera, se construyó otra variable dicotómica para el caso de las revistas, que podía tomar esos mismos valores si estas se encontraban en acceso abierto o no.

Como complemento a lo anterior, se definió una regla de decisión para que el algoritmo asigne la categoría de ruta dorada en el caso de que ambas columnas tengan el valor de verdadero. Esta misma decisión se toma en caso de que la revista no sea de acceso abierto, pero una columna adicional, denominada "best_oa_location”, incluya el DOI del respectivo artículo.

4. Depuración de la información

Posteriormente, se utiliza un algoritmo ${ }^{19}$ para asignación de las rutas de acceso abierto para cada uno de los artículos disponibles en la base de datos, en caso de tener este criterio, o bien, indicar si es de acceso cerrado. Gracias a lo anterior, y combinando bases de datos, se identificaron las siguientes columnas de metadatos:

- DOI, indicando el DOI del artículo

- Open_Access, indicando si la revista es de acceso abierto

- OA_Green, indicando si el artículo es de ruta verde

- $O A \_G o l d$, indicando si la revista es de acceso dorado

- OA_Gold_Article, indicando si se cumple la segunda condición del apartado anterior para ser acceso dorado

- $I S S N$, a partir de la revista

- ISSNWOS, a partir de la base de datos de WOS

\section{Identificación del pago APC}

A partir de los artículos publicados en ruta dorada o ruta verde, es posible identificar si hubo pago de APC relacionado a estos. Para ello, se toma el ISSN de cada una de las revistas y este se cruza con la plataforma DOAJ, la cual brinda información sobre los cobros, en moneda local de la revista, del APC que solicita cada revista para publicar los artículos. Esto permite complementar la base de datos, agregando las columnas APC, OAGISSN (que corresponde al ISSN realmente utilizado en el archivo), además se convierten los datos de moneda local en dólares, utilizando el módulo Currency Convertir de Python.

\section{Indicadores de visibilidad}

Finalmente, para cada artículo se descargan indicadores de impacto científico que han alcanzado. Esto se logra identificando las citas de Google Scholar (GS) con las siguientes variables: número de citas, enlace a las citas, perfil en GS de los autores y otros metadatos complementarios.

Gracias a estos seis pasos, fue posible presentar un diagnóstico a la situación de la Universidad de Antioquia frente al acceso abierto, a partir de los siguientes ítems: producción en acceso abierto, tipos de acceso, APC y el Openness Index; y a su vez, evidenciar las características y metodología del Modelo propuesto, que permite a cualquier universidad, (ojalá especialmente de América Latina, por todas sus implicaciones de acceso al 
conocimiento y de costos-sobrecostos), identificar su situación general frente al acceso abierto. El algoritmo que permitió el desarrollo de la investigación y consolidar la base de datos, se realizó en Colaboratory, extensión de Google Drive que permite trabajar con Notebooks de Python ${ }^{20}$.

\section{RESULTADOS Y DISCUSIÓN}

Mediante la extracción de información realizada de las bases de datos, se obtuvo una descripción de la producción de la UdeA en Acceso Abierto. A partir del trabajo de Nichols y Twidale (2017), la siguiente sección se organiza en dos subsecciones: descripción general de la producción en acceso abierto y evolución de las métricas de apertura de la institución.

\subsection{Descripción de la producción científica de la UdeA en Acceso Abierto}

\section{¿Cuánto ha sido la producción de la UdeA?}

En este estudio se identificaron 15504 (7990 artículos analizados, al tener DOI), realizados por la UdeA, con una trayectoria de producción que data desde 1899 (fue fundada en 1803) y que tuvo un crecimiento exponencial a partir de la primera década del siglo XXI. Este crecimiento puede estar explicado por los cambios de política de incentivos y de organización del sistema de ciencia y tecnología, tanto a nivel nacional como institucional, que se han dado desde 1990 (Maz-Machado, Jiménez-Fanjul y Villarraga Rico, 2016). La Figura 3 muestra el crecimiento de la producción de la Universidad, con dos sub-divisiones: acceso abierto y acceso cerrado ${ }^{21}$.

FIGURA 3

Publicaciones de la UdeA: Producción total (a) y porcentaje de acceso abierto vs cerrado (b)

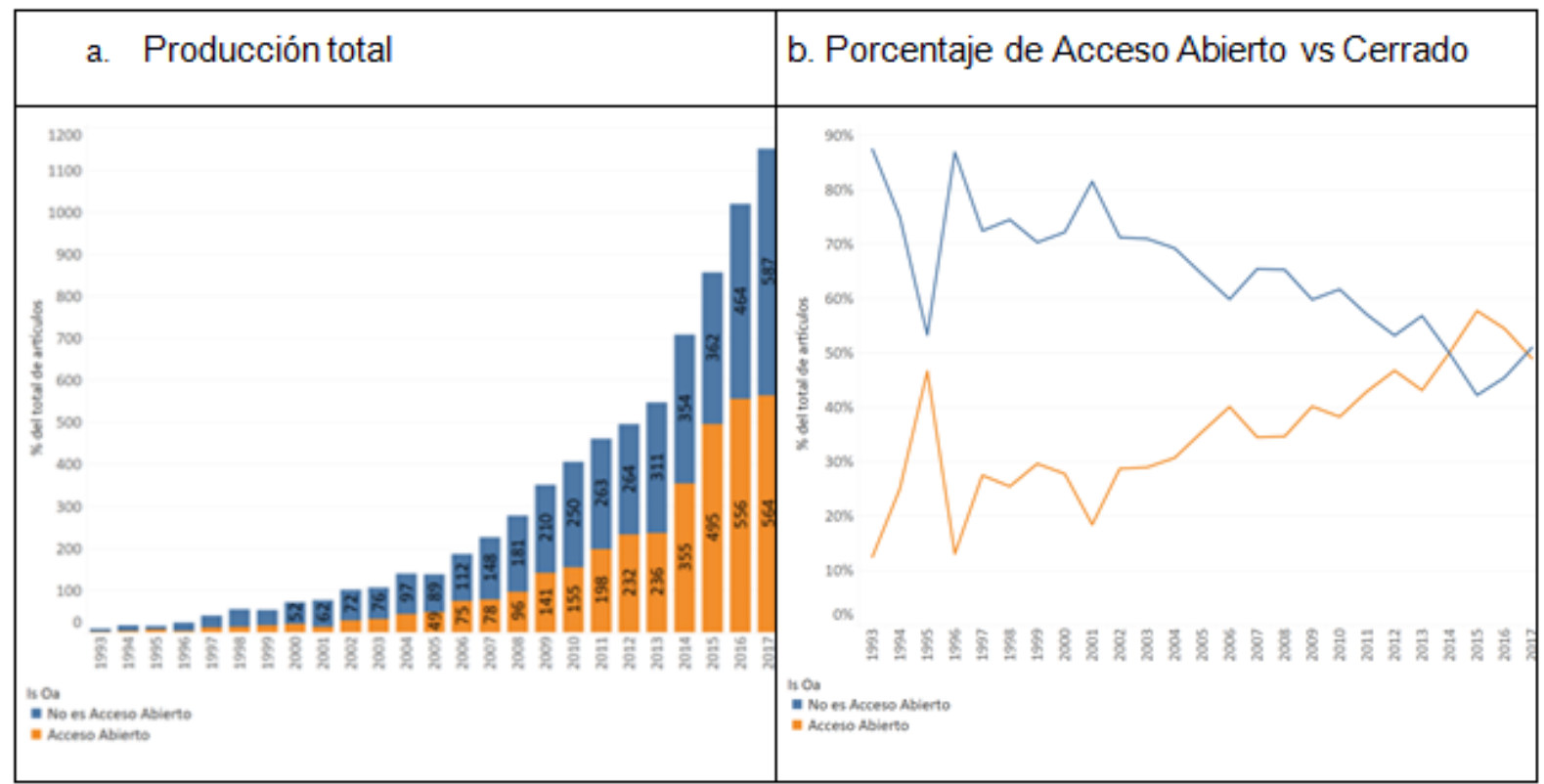

Fuente: elaboración propia

Como se vio en la sección de contenido teórico, no hay una única tipología de acceso a las revistas, sino que pueden diferenciarse cuatro grandes variantes (dorada, verde, híbrida y cerrada). En la figura 4 puede observarse la distribución de la producción en acceso abierto de la UdeA por tipo de acceso, tomando como 
referencia tres momentos temporales con suficiente producción: 2000, que representa las publicaciones entre 2000 y 2009; 2010, que representa entre 2010 y 2015; y 2017, que representa las publicaciones para ese año. Allí se vislumbra el crecimiento: 27,78\% en 2000, 38.27\% en 2010 y 49\% en 2017. Es importante destacar que este desempeño se ha dado en medio de un contexto en el que la UdeA no ha generado incentivos explícitos para la producción en acceso abierto, por lo que el crecimiento porcentual surge a partir de los intereses/necesidades propias de los investigadores y las prácticas de las comunidades académicas en las que se desenvuelven.

FIGURA 4

Distribución de la producción de la Universidad de Antioquia en el tiempo

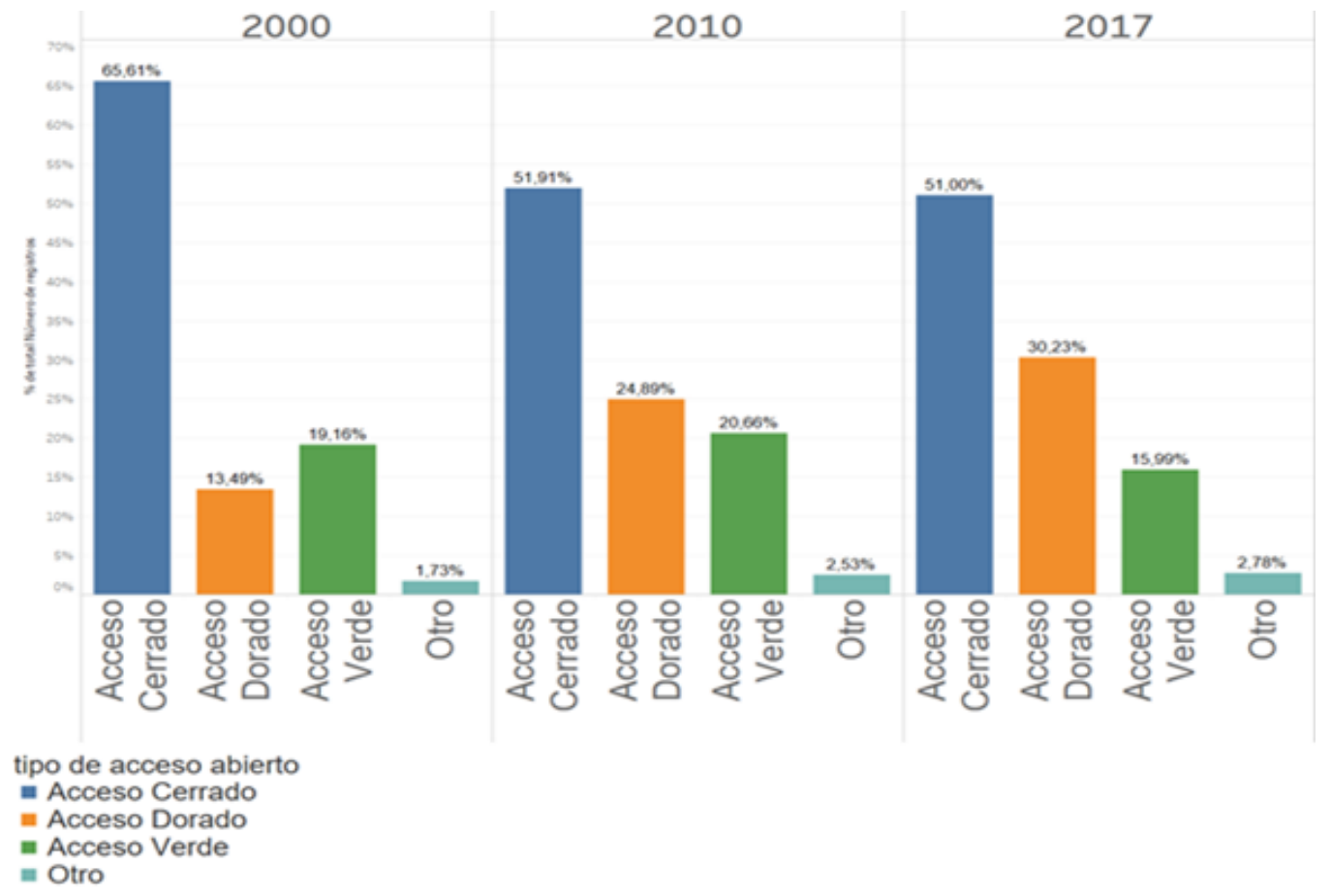

Fuente: elaboración propia

Esta figura indica, además, el incremento en publicaciones de acceso abierto que ha presentado la Universidad de Antioquia en los últimos años, tanto en acceso verde como en acceso dorado. Se observa que en una primera fase las publicaciones de acceso cerrado eran dominantes pero existía una importante fracción de artículos publicados en la vía verde. En los dos periodos siguientes, es notable el incremento que ha tenido la vía dorada como patrón de publicación de la UdeA, alcanzando a la vía verde en el primer lustro de la presente década y superándola para 2017, incluso acercándose a la publicación cerrada.

En este sentido, se puede considerar que la UdeA apunta a que en los próximos años la vía dorada sea su principal canal de comunicación científica, lo que ha de ser considerado, y discutido, para la toma de decisiones de política de investigación. Así, analizando los datos de distribución de la producción en acceso abierto de la UdeA se puede confirmar esta posible tendencia. En la década de los noventa se publicaba un $77 \%$ de la producción abierta en la vía verde con un $19 \%$ en vía dorada ${ }^{22}$. En la década siguiente, se dio una transformación en la medida que la misma proporción pasó a un 56\% y un 39\% respectivamente. La composición dio el giro en la primera parte de esta década, 2010-2015, en la medida que la ruta verde pasó a pesar un 43\% mientras que la dorada un 52\%. Para 2017 la composición correspondía a 33\% y 62\% 
respectivamente, lo que da cuenta de que la forma de comunicación abierta de la investigación universitaria ha dado un giro radical.

Finalmente, esto no aplica solo para la producción abierta de la UdeA, sino que es un patrón general: a la fecha el $23 \%$ del total de la producción se realiza en la vía dorada. Pero este porcentaje ha implicado un avance progresivo de la vía en la UdeA, desde un 13\% en los 2000, a un 25\% en el primer lustro, y un 30\% en 2017. Este crecimiento da cuenta de que la Universidad no ha sido ajena a la tendencia general de la comunicación científica. A su vez, a pesar de la limitación de los datos, por el DOI, si se aplica el Openness Index, la UdeA, para ese $51,5 \%$ de su producción analizada, alcanzaría un 0,44; aunque sería mucho mayor considerando que buena parte de la producción que no tenía DOI era procedente de SciELO y RedALyC (de años anteriores al posicionamiento del DOI), las cuales son plataformas latinoamericanas de origen y trayectoria en acceso abierto, y mayoritariamente sin pago de APC.

\section{¿En cuáles revistas produce la UdeA?}

Bajo los parámetros de este estudio, la UdeA ha escrito en más de 2000 revistas, tanto nacionales como internacionales, de las cuales el $45 \%$ publican en acceso abierto. La figura 5 presenta las revistas en las cuáles hay mayor publicación por parte de la UdeA, donde se puede observar que 8 de las 11 revistas publican documentos en acceso abierto. Asimismo, se observa un peso significativo de la revista Biomédica, lo que da cuenta de una alta comunicación de la UdeA sobre los problemas de salud de carácter más local.

FIGURA 5

Revistas con mayor producción científica de la Universidad de Antioquia

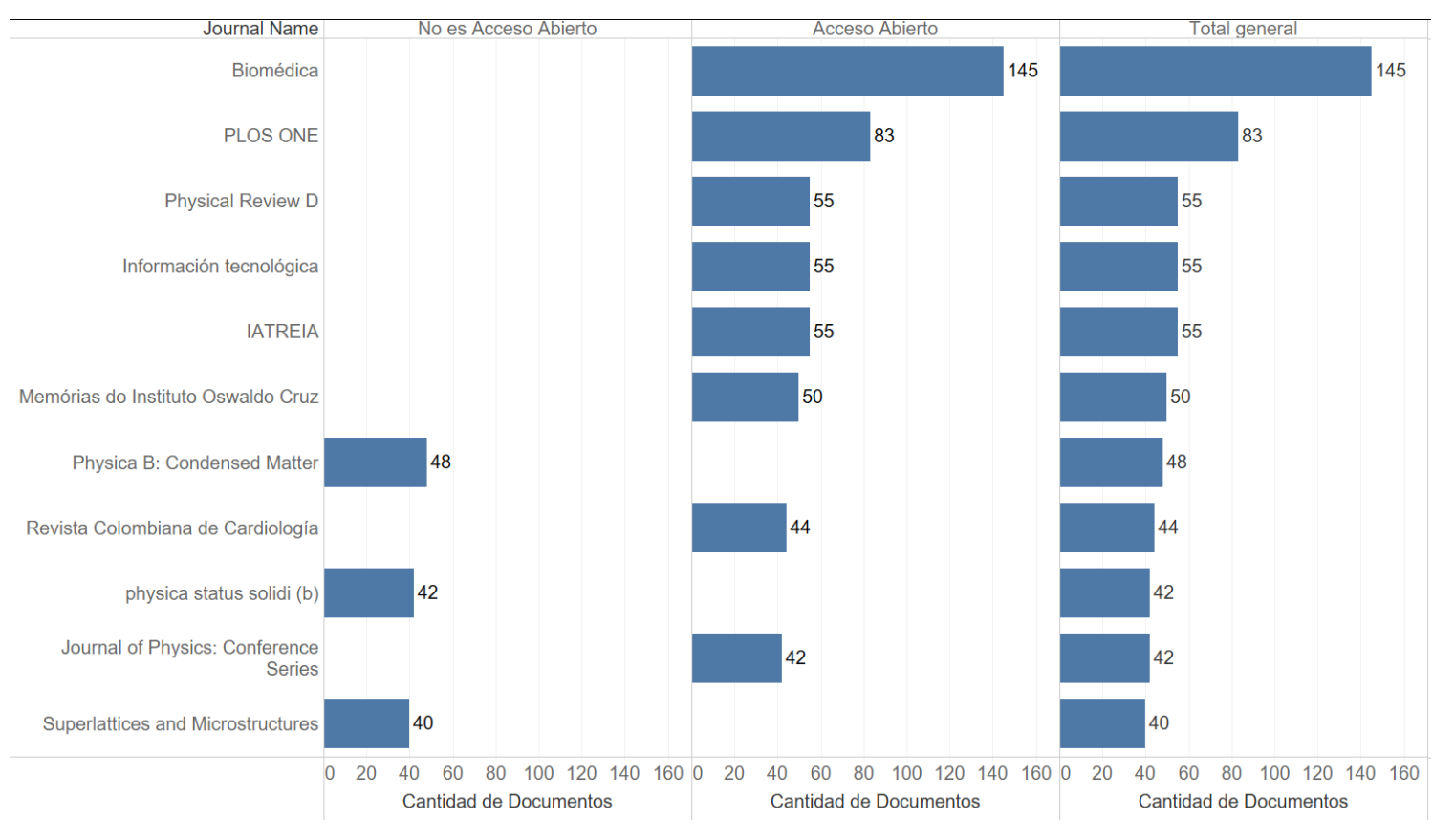

Fuente: elaboración propia

\section{¿Cuánto se ha pagado por los artículos de la UdeA?}

En la sección de metodología se especificó el procedimiento para encontrar el gasto en APC que se ha hecho por las publicaciones que realiza la Universidad. En este punto es importante recordar que no necesariamente estos gastos los realizó sólo la UdeA, sino que se hicieron en marco de las publicaciones de la 
Institución. Esto porque la base de datos no identifica quién(es) pagó el artículo sino cuánto cobra la revista, por lo que el pago pudo realizarlo alguna de las instituciones participantes de la publicación o en diferentes proporciones.

Las siguientes figuras muestran la evolución de los pagos que se han realizado en el tiempo: en la figura 6 se muestra el valor absoluto del gasto en dólares por editorial, mientras que en la 7 señala el porcentaje que tiene cada editorial en el año respectivo. En conjunto, el gasto que se ha realizado es cercano a USD un millón de dólares. En este sentido resulta claro el avance que ha tenido el pago APC en la UdeA, puesto que en el año 2010 se pagaron alrededor de USD 30.000, mientras que en 2017 se pagó cerca de USD 180.000, lo que implica un crecimiento de 6 veces en 7 años.

La editorial a la que se le han pagado más recursos es Springer Nature, con un porcentaje promedio superior al 30\% en la presente década. En segundo lugar, se encuentra PLOS con un promedio del $21 \%$ en el mismo rango de tiempo. Luego, Frontiers Media S.A, en la que se ha invertido el 12\% de los recursos. En cuarto lugar, MDPI AG con 9\% y luego, Elsevier BV con un $6 \%$ de los recursos. En conjunto, estas cinco editoriales acumulan el $77 \%$ de los recursos que se han pagado en APC, por publicaciones realizadas por la Universidad.

\section{FIGURA 6}

Valor absoluto del gasto en dólares por editorial

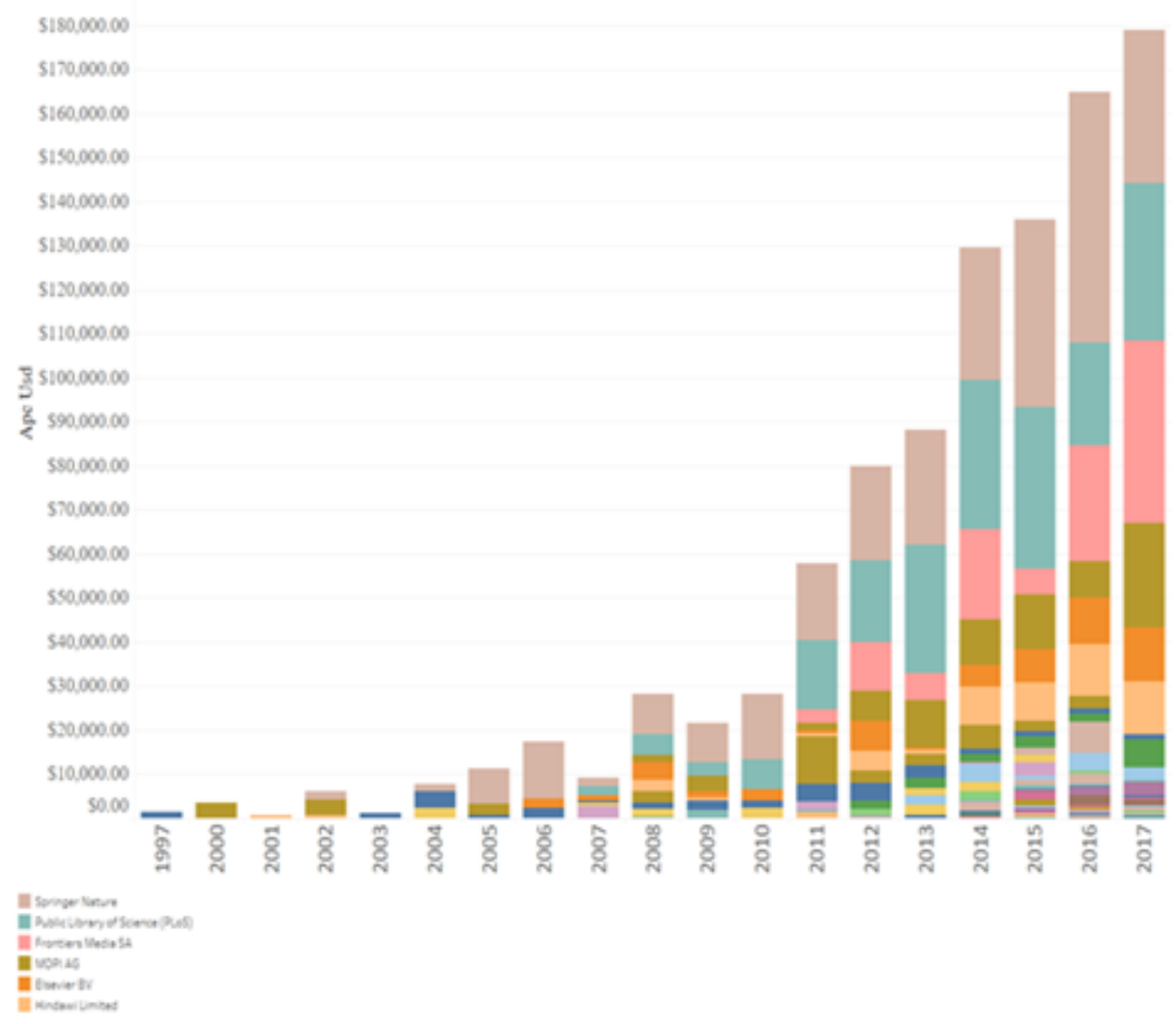

Fuente: elaboración propia 
FIGURA 7

Porcentaje que tiene cada editorial en el año respectivo

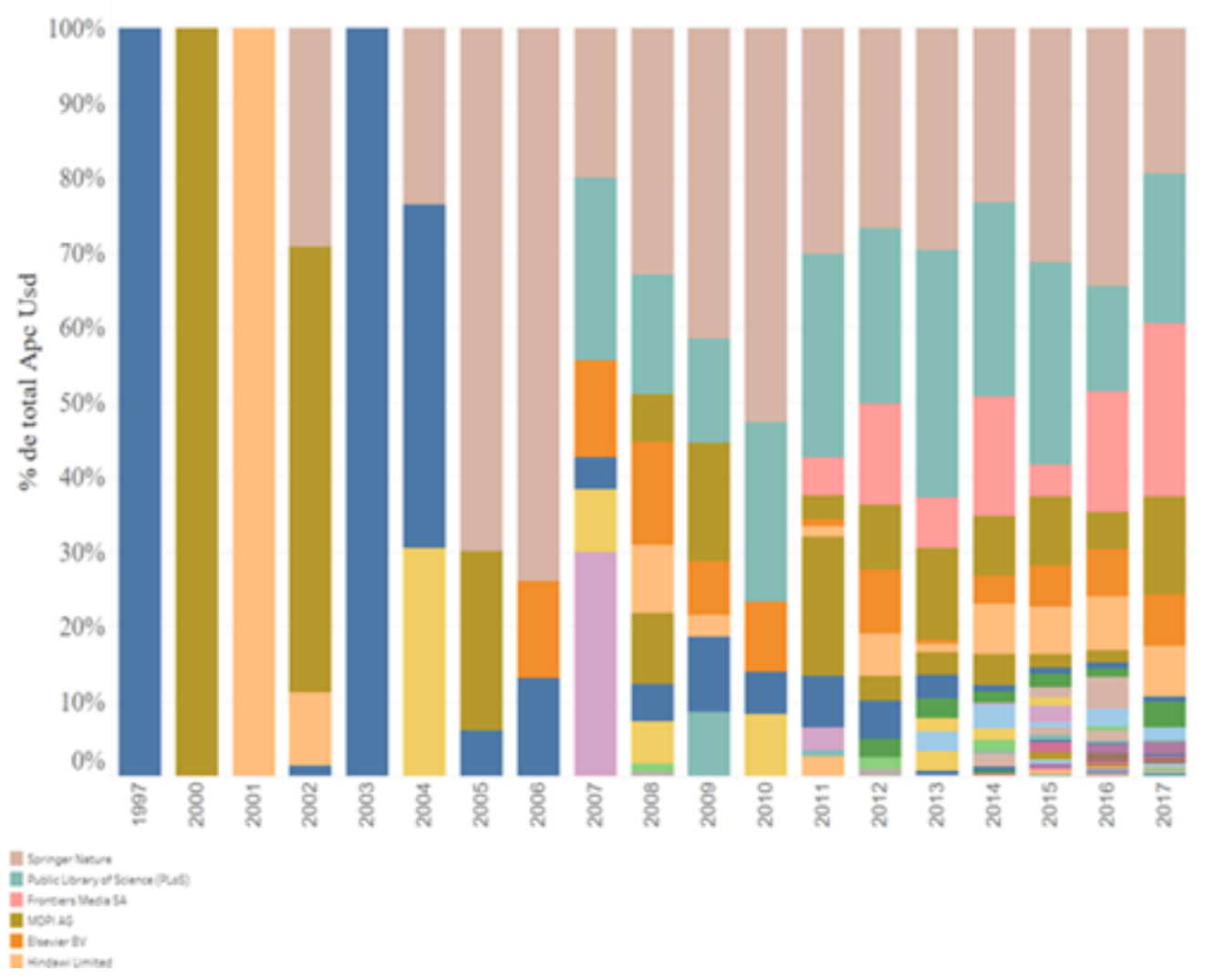

Fuente: elaboración propia

\section{¿En qué idiomas se publica en acceso abierto en la UdeA?}

Un aspecto positivo de la publicación de la Universidad en acceso abierto es que tiene mayor diversidad idiomáticaque cuando se publica en acceso cerrado. La diferencia es significativa, casi un $92 \%$ de las publicaciones en acceso cerrado se publican en inglés, mientras que un $61 \%$ de acceso abierto se publica en ese idioma. El salto lo dan las publicaciones en español, que pesan casi un 34\% en las publicaciones en acceso abierto, lo cual puede tener varias explicaciones posibles: 1) las bases en acceso abierto incluyen a SciELO y RedALyC, cuya fuerte presencia de revistas latinoamericanas implican una mayor presencia de la publicación en español; por otro lado, 2) las revistas en acceso cerrado pueden pertenecer a WoS o SCOPUS, donde distintos estudios han dado cuenta de sus sesgos idiomáticos.

\section{¿Cuáles son las editoriales con mayor influencia en la UdeA?}

En el caso de las revistas de acceso cerrado se observa una dependencia significativa en la editorial Elsevier, dado que el 39\% de los artículos de esta tipología producidos desde la UdeA se han realizado en revistas de esta editorial. Esto puede observarse en la figura 8 donde las revistas Elsevier (de color naranja) sobresalen frente al resto de editoriales. En segundo lugar, se encuentran las revistas de Springer Nature con el 10,77\% mientras que las de Wiley pesan el 8,55\%. En esta medida, tres editoriales de pago publican el 59\% de la producción científica de la UdeA, lo que decanta una dependencia para acceder a su propia producción científica y la necesidad de la institución en renegociar con estas editoriales. Si se considera el conjunto de 
la producción institucional, se infiere que la UdeA pagaría a tres editoriales para acceder al $61,53 \%$ de toda la investigación que realiza con recursos públicos, por lo que se refleja la necesidad de discusión desde estas cifras, y la importancia de que las universidades apuesten por el acceso abierto en su producción.

Por otro lado, la amplia diversidad de colores de la parte derecha de la gráfica, que muestra la producción en acceso abierto, da cuenta de que en este tipo de publicación se reduce la dependencia de la Universidad de Antioquia a una única editorial. Cada una de las cuatro revistas en las que más publica la UdeA en acceso abierto son de tres editoriales distintas (Instituto Nacional de Salud, PLOS, Universidad de Antioquia), lo cual es un valor significativo e incluso estratégico para las actividades de la UdeA.

\section{FIGURA 8}

Editoriales con mayor número de publicaciones de la UdeA

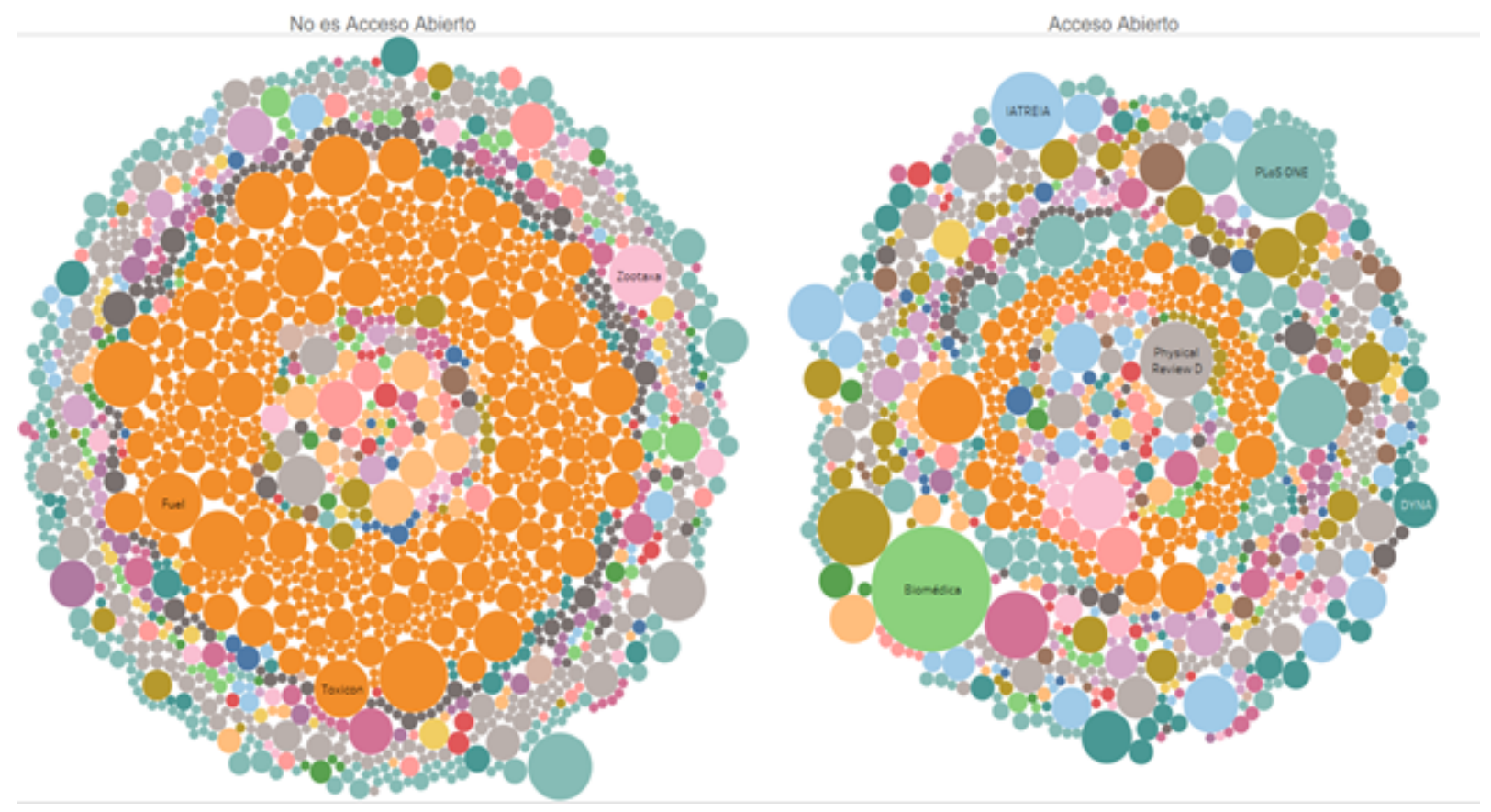

Fuente: elaboración propia

\subsection{Evolución de las métricas de apertura de la institución}

\section{¿Cómo ha sido el desempeño de las citas de los artículos de la UdeA?}

A partir de la base de datos utilizada para el presente estudio, se identificó un total de 148.965 citas a los artículos, de las cuales $73.206(49,14 \%)$ son para los artículos de acceso abierto, mientras que 75.759 $(50,86 \%)$ son para los artículos que son en acceso cerrado. Ahora bien, para analizar cuál de los dos esquemas de publicación tiene mayor impacto científico, entendiendo impacto como la cantidad de citas (Mingers y Leydesdorff, 2015), es más adecuado utilizar la cantidad de citas por artículo. La tabla 1 presenta un resumen descriptivo del comportamiento de las citas, mientras que la figura 9 presenta un gráfico de cajas y bigotes mostrando la diferencia de desempeño. 
TABLA 1

Comportamiento de citas en acceso abierto y no abierto de la producción UdeA

\begin{tabular}{|l|c|c|}
\hline \multicolumn{1}{|c|}{ Indicador } & Acceso Abierto & Acceso no Abierto \\
\hline Documentos & 3466 & 4524 \\
\hline Cantidad de citas & 73.206 & 75.759 \\
\hline Promedio de citas por documento & 21,121 & 16,746 \\
\hline Valor Máxımo & 5458 & 1532 \\
\hline Mediana de Citas & 2 & 5 \\
\hline Desviación Estándar de Citas & 143,1 & 40,1 \\
\hline
\end{tabular}

Fuente: elaboración propia

\section{FIGURA 9}

Diferencia de desempeño de citas entre acceso cerrado y acceso abierto de la producción UdeA
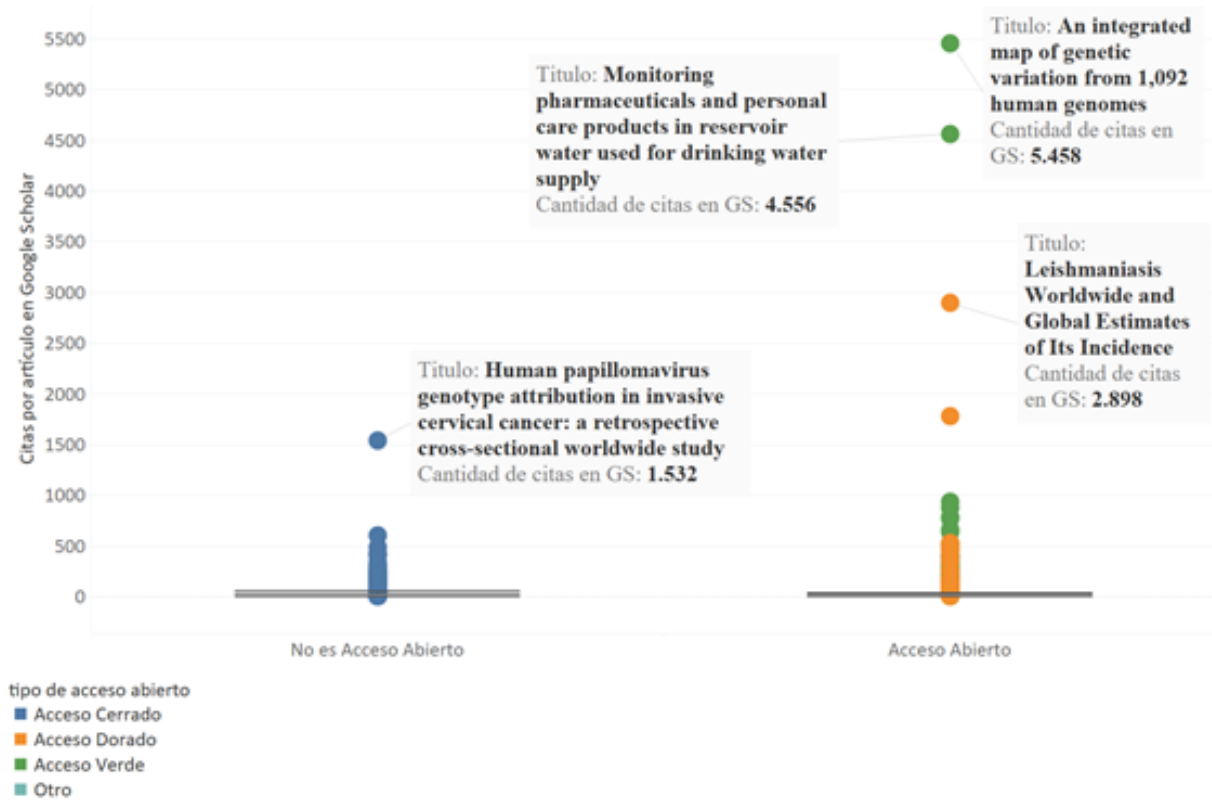

Fuente: elaboración propia

Esta información permite identificar algunas perspectivas interesantes. En primer lugar, se confirma que la publicación en acceso abierto permite obtener mayor cantidad de citas para los artículos publicados, con casi un $30 \%$ más de citas por artículo para ese grupo poblacional. Sin embargo, esta mayor posibilidad no se distribuye uniformemente en la población, puesto que indicadores generales de dispersión (como la desviación estándar) es significativamente mayor en los productos de acceso abierto que en los que no son de acceso abierto y, asimismo, la mediana es mayor en los que no son de acceso abierto que en los que sí se publican bajo este criterio. Lo anterior se confirma con el gráfico de cajas y bigotes, puesto que se observa que hay una mayor cantidad de artículos con una cantidad atípica de citas en la población de acceso abierto, mientras que hay mayor uniformidad en los artículos de acceso cerrado.

\section{CONCLUSIONES Y RECOMENDACIONES}

Se han presentado los datos específicos para la Universidad de Antioquia, además de la metodología propuesta (Modelo) para identificar la situación en "Acceso Abierto" de una universidad, que puede ser 
replicable en cualquier institución que quiera identificar su situación frente a este tema, identificando sus debilidades y fortalezas, y definiendo sus esfuerzos a futuro (a corto, mediano y largo plazo), para buscar un mayor índice de acceso abierto, y desde este componente, impulsar los otros componentes de la ciencia abierta (Uribe-Tirado y Ochoa, 2018 a,b).

A partir de esto, se exponen a continuación, las siguientes conclusiones buscando dar una mirada particular desde la Universidad de Antioquia, pero generalizable a otras universidades que quieran seguir esta metodología:

- Aunque los datos presentados en este artículo generan una limitación porque la metodología considera solo la producción con DOI, la tendencia de un $51,5 \%$ de toda la producción da una importante caracterización. Incluso, como se indicó, al identificar un Openness Index del 0,44, estos datos de acceso abierto en la UdeA serían mayores, considerando que una buena parte de nuestra producción está en esta modalidad, aunque carezca de DOI en años anteriores ${ }^{23}$. Se considera que, en próximos análisis, ya que es una estrategia de medición que se va a continuar en la institución de manera continua, no constituirá mayores problemas gracias a que el DOI es una realidad cada vez más presente en la producción científica desde América Latina.

- En el acceso cerrado hay una concentración de la publicación en las revistas de Elsevier, en cambio cuando se trata de revistas de acceso abierto hay mayor diversidad de editoriales. En esta medida se considera que el promover la publicación en acceso abierto reduce la dependencia de la universidad a las grandes editoriales y permitiría disminuir los pagos que realiza para acceder a su propio conocimiento.

- Los investigadores de la UdeA autónomamente han aumentado su producción en acceso abierto, incluso en un contexto de cero incentivos institucionales. En este sentido, vale la pena pregunta, ¿por qué lo han hecho? Y se incentivaran, ¿cuánto crecería a corto y mediano plazo? considerando la apuesta institucional por el acceso abierto, por la ciencia abierta.

- La publicación en ruta dorada, en su variante híbrida que implica pago de APC, está ganando espacio progresivamente como el principal canal de comunicación de la ciencia en la Universidad de Antioquia. Es necesario, entonces, que la institución, discuta y tome medidas de política investigativa para afrontar este fenómeno (fomentarlo, regularlo, etc.)

- No obstante, por experiencias en otras universidades y recomendaciones de organizaciones de ciencia, acordes con la ciencia abierta, es necesario integrar los mandatos a publicar en el repositorio institucional ${ }^{24}$, con los requisitos de investigación (términos de referencia de convocatorias) o con incentivos por la publicación preferentemente en el acceso abierto (premios, prioridad en trámites investigativos, puntos adicionales en convocatorias, etc.), además de la formación continua a los investigadores (tratando de aclarar muchos mitos) ${ }^{25}$; para que así la proporción de textos depositados en el Repositorio sea cada vez mayor, ya que, como hemos señalado en otros trabajos:

El lugar preferencial de registro de la producción científica de una universidad debe ser su repositorio, ya que es su propio sistema de información, es donde todo el público puede consultar y acceder a su producción, y así responder más fácil la pregunta: ¿Qué ha publicado la Universidad de los últimos años?, y no tener que ir de base de datos en base de datos, muchas de ellas, sin posibilidad de acceso, pues implican pago de altos costos (Uribe-Tirado, 2015, p.5).

\section{REFERENCIAS BIBLIOGRÁFICAS}

Abadal, E. (2012). Acceso abierto a la ciencia. Barcelona: Editorial UOC. Recuperado de https://core.ac.uk/downloa d/pdf/11889005.pdf 
Anglada, L., y Abadal, E. (2018). ¿Qué es la ciencia abierta? Anuario ThinkEPI, 12, 292-298. Recuperado de https:// recyt.fecyt.es/index.php/ThinkEPI/article/view/thinkepi.2018.43/39164

Briceño, Y. (2013). El modo emergente de la comunicación de la ciencia: incidencias y gestión distribuida en América Latina (Tesis Doctoral). Universidad de Los Andes (Venezuela). Recuperado de http://eprints.rclis.org/28500/

Budapest Open Access Initiative (2002). Budapest Open Access Initiative. Recuperado de https:// www.budapestopenaccessinitiative.org/read

Declaración de Bethesda (2003). Declaración de Bethesda sobre publicación en acceso abierto. Recuperado de https://i ctlogy.net/articles/bethesda_es.html

Declaración de Berlín (2003). Declaración de Berlín sobre el acceso abierto. Max Planck Society. Recuperado de https ://openaccess.mpg.de/Berlin-Declaration

Debat, H., y Babini, D. (2019). Plan S en América Latina: Una nota de precaución (pre-print). Recuperado de: https ://zenodo.org/record/3332621 [Título original Plan S in Latin America: A precautionary note].

García Martín, M. (2015). Las revistas de Geografía en el Journal Citation Reports: lucro económico versus acceso abierto. Revista española de documentación cientifica, 38(4). Recuperado de https://idus.us.es/xmlui/handle/11 $441 / 55301$

Gibbons, M. (2000). Mode 2 society and the emergence of context-sensitive science. Science and Public Policy, 27 (3), 159-163. Recuperado de https://academic.oup.com/spp/article/27/3/159/1650494

González, V. H., Reverter-Masia, J., y Jové-Deltell, C. (2017). Producción científica de los profesores del área de Educación Física y deportiva en Cataluña (quinquenio 2005-2009 versus 2010-2014). SPORT TK-Revista EuroAmericana de Ciencias del Deporte, 6(1), 17-24. Recuperado de https://revistas.um.es/sportk/article/view 1280361

Harnad, S., Brody, T., Vallières, F., Carr, L., Hitchcock, S., Gingras, Y., Oppenheim, C., Hajjem, C., y Hilf, E. R. (2008). The access/impact problem and the green and gold roads to open access: An update. Serials review, 34(1), 36-40. http://dx.doi.org/10.1080/00987913.2008.10765150

Hook, D.W., Calvert, I., y Hahnel, M. (2019). The Ascent of Open Access. An analysis of the Open Access landscape since the turn of the millennium. Recuperado de https://digitalscience.figshare.com/articles/The_Ascent_of_Open_ Access/7618751

Koskinen, K., Lappalainen, A., Liimatainen, T., Nevalainen, E., Niskala, A.,y Salminen, P. J. (2010). The current state of Open Access to research articles from the University of Helsinki. ScieCom Info. Recuperado de https://held a.helsinki.fi/bitstream/handle/10138/36901/ScieComInfo.pdf?sequence $=6$

Laakso, M., y Björk, B. C. (2013). Delayed open access: An overlooked highimpact category of openly available scientific literature. Journal of the American Society for Information Science and Technology, 64(7), 1323-1329. h ttps://doi.org/10.1002/asi.22856

Larivière, V., Haustein S., y Mongeon P. (2015). The Oligopoly of Academic Publishers in the Digital Era. PLoS ONE, 10(6), e0127502. https://doi.org/10.1371/journal.pone.0127502

López-Borrull, A. (2012). Física vs química: dos modelos de publicación científica. El profesional de la información, 21(2), 167-172. Recuperado de http://eprints.rclis.org/18736/

Luchilo, L.J. (2019). Revistas científicas: oligopolio y acceso abierto. Revista Iberoamericana de Ciencia, Tecnologia y Sociedad-CTS, 14(40). Recuperado de: http://www.revistacts.net/files/Volumen_14_Numero_40/02Luchilo .pdf

Maz-Machado, A., Jiménez-Fanjul, N. N., y Villarraga Rico, M. E. (2016). La producción científica en SciELO: un análisis bibliométrico. Revista Interamericana de Bibliotecología, 39(2), 111-119. http://dx.doi.org/10.17533/u dea.rib.v39n2a03

Mingers, J., y Leydesdorff, L. (2015). A review of theory and practice in Scientometrics. European Journal of Operational Research, 246(1), 1-19. https://dx.doi.org/10.1016/j.ejor.2015.04.002

Morrison, H., Salhab, J., Calvé-Genest, A., y Horava, T. (2015). Open access article processing charges: DOAJ survey. Publications, 3(1), 1-16. Recuperado de https://www.mdpi.com/2304-6775/3/1/1 
Nichols, D. M., y Twidale, M. B. (2017). Metrics for openness. Journal of the Association for Information Science and Technology, 68(4), 1048-1060. https://dx.doi.org/10.1002/asi.23741

Piwowar, H., Priem, J., Larivière, V., Alperin, J. P., Matthias, L., Norlander, B., Farley, A., West, J., y Haustein, S. (2018). The state of OA: a large-scale analysis of the prevalence and impact of Open Access articles. PeerJ, 6, e4375. http://dx.doi.org/10.7717/peerj.4375

REBIUN (2019). Recomendaciones sobre la monitorización del acceso abierto. Recuperado de http://213.32.37.214/x mlui/handle/20.500.11967/245

Resnick, B., y Belluz, J. (2019). The war to free science. En Vox. Recuperado de: https://www.vox.com/the-highlight /2019/6/3/18271538/open-access-elsevier-california-sci-hub-academic-paywalls

Schiermeier, Q., y Rodriguez Mega E. (2017). Scientists in Germany, Peru and Taiwan to lose access to Elsevier journals. Nature News, 541(7635). http://dx.doi.org/10.1038/nature.2016.21223

Siso-Calvo, B., y Arquero-Avilés, R. (2018). Acceso abierto, promoción y difusión digital de proyectos I+ D+ i: el caso de la Universidad Complutense de Madrid. Ibersid, 12(2). Recuperado de http://ibersid.eu/ojs/index.php/ibe $\mathrm{rsid} /$ article/view/4565/3931

Suber, P. (2008). Gratis and libre open access. SPARC Open Access Newsletter, 124. Recuperado de https://dash.har vard.edu/handle/1/4322580

Tennant, J.P., Crane, H., Crick, T., Davila, J., Enkhbayar, A., Havemann, J., Kramer, B., Martin, R., Masuzzo, P., Nobes, A., Rice, C., Rivera-López, B.S., Ross-Hellauer, T., Sattler, S., Thacker, P., y Vanholsbeeck, M. (2019) Ten myths around open scholarly publishing. PeerJ Preprints, 7, e27580v1. https://doi.org/10.7287/peerj.pre prints.27580v1

Toledo, E. G. (2014). Imposturas en el ecosistema de la publicación científica. Revista de Investigación educativa, 32(1), 13-23. https://doi.org/10.6018/rie.32.1.190251

Uribe-Tirado, A. (2015). Acceso abierto y calidad de las publicaciones. Mitos y realidades. En Semana Internacional del Acceso Abierto - Universidad de Antioquia, octubre. Recuperado de http://www.openaccessweek.org/events /jornadas-por-el-acceso-abierto-universidad-de-antioquia-2005?xg_source=activity

Uribe-Tirado, A., y Ochoa-Gutiérrez, J. (2018a). Perspectivas de la ciencia abierta. Un estado de la cuestión para una política nacional en Colombia. BiD: textos universitaris de biblioteconomia i documentació, 40. https://dx.doi.o $\mathrm{rg} / 10.1344 / \mathrm{BiD} 2018.40 .5$

Uribe-Tirado, A., y Ochoa-Gutiérrez, J. (2018b). Ciencia abierta y bibliotecas académicas: una revisión sistemática de la literatura. Iris - Informação, Memória e Tecnologia, 4(1), 49-69. Recuperado de https://periodicos.ufpe.br/r evistas/IRIS/article/view/238911

\section{Notas}

1 Este artículo es derivado de la investigación: "Impacto social, cientifico y actividades de vinculación con el entorno de centros de investigación de la Universidad de Antioquia" que fue financiada por la convocatoria Programática de Ciencias Sociales y Humanidades de la Universidad de Antioquia (Medellín-Colombia), año 2018.

2 La UdeA, es la segunda universidad colombiana en este ítem, según distintos indicadores. Ver: https://www.scimagoir.com/ institution.php?idp=1906; https://bit.ly/2ovSLpp; http://www.udea.edu.co/wps/portal/udea/web/inicio/institucional/data-u dea/investigacion/proyectos

3 Se les considera oligopolios en la medida que las grandes casas editoriales son las dueñas de las revistas, las bases de datos para acceder a ellas, y de las plataformas para medir la citación como criterio de calidad académica

4 Según Anglada y Abadal (2018, p. 294):

El acceso abierto a los contenidos académicos ha precedido al concepto de ciencia abierta y, quizá, ha condicionado algo su percepción. Si bien tenemos la tendencia de asimilar acceso abierto solamente a los artículos, en el contexto de la ciencia abierta, lo que debe estar en abierto es cualquier resultado de la investigación (artículos y datos) así como los instrumentos 
auxiliares usados, como pueden ser los cuadernos de laboratorio. Del mismo modo que lo era para artículos, aquí el significado de "abierto" es doble: gratuito y libre.

5 En la siguiente línea del tiempo pueden verse diferente hitos: https://www.preceden.com/timelines/288283 (en color azul están los correspondientes al acceso abierto)

6 Si se toman los datos de DOAJ de revistas sin APC, se puede identificar que la mayoría de revistas de acceso abierto son de origen universitario: https://bit.ly/1O5U4eQ

7 Si se consideran los datos de OpenDOAR, se puede identificar que la mayoría de los repositorios son también de este origen: h ttps://bit.ly/2M7wGqS - https://bit.ly/2JHOIyx

8 Aunque no existe una plataforma que reúna los repositorios temáticos de manera específica, si se revisan recursos como Disciplinary repositories, es posible identificar el origen universitario de estos recursos de información: http://oad.simmons.edu/ oadwiki/Disciplinary_repositories

9 Ante este nuevo cobro, han surgido propuestas para hacerlo visible y generar discusión sobre estos altos costos, y que están asumiendo en buena parte las universidades. Una de las más significativas es Open APC initiative: https://treemaps.intact-proje ct.org/apcdata/openapc/

10 Ver diferentes casos: https://universoabierto.org/?s=elsevier (Consultado: 19-3-2019)

11 Para mayor información sobre esta iniciativa, por favor visitar: https://consortia.com.co/

12 Los trabajos reseñados fueron seleccionados tras un rastreo amplio de lo que se ha publicado en acceso abierto como parte de la ciencia abierta, a partir de trabajos investigativos anteriores (Uribe-Tirado y Ochoa-Gutiérrez, 2018a, 2018b).

13 Ver diferentes rutas: Guest Post: From Supermarkets to Marketplaces - The Evolution of the Open Access Ecosystem https://schola rlykitchen.sspnet.org/2018/05/14/guest-post-supermarkets-marketplaces-evolution-open-access-ecosystem/?informz=1 - Las 5 rutas para llegar al acceso abierto: verde, dorada, bronce, hibrida y diamante. https://universoabierto.org/2019/03/13/las-5-rutas -para-llegar-al-acceso-abierto-verde-dorada-bronce-hibrida-y-diamante/

14 Los resultados de este trabajo, se conectarían años después con la Open APC Initiative 2018-2019 (https://treemaps.intact-pr oject.org/apcdata/openapc/) y los sorprendentes y altos gastos que está implicando.

15 Los trabajos desde SciELO y RedALyC, no entran directamente en el rastreo ya que una muestra representativa no poseía DOI. No obstante, sí se tiene en cuenta Scielo Citation Index, el cual recoge una buena parte de la producción de SciELO. Respecto a RedALyC, una buena cantidad de sus trabajos, se encuentran duplicados en las otras bases de datos consideradas en el estudio. En las conclusiones, se menciona como parte de las limitaciones y un aspecto que se solucionará en próximos estudios, que ya en los últimos años, el DOI es una realidad cada vez más frecuente para artículos indexados en SciELO y RedALyC, por lo que, a futuro, no será problema desde la metodología propuesta.

$16 \mathrm{El}$ algoritmo está disponible en: https://nbviewer.jupyter.org/github/restrepo/medicion/blob/master/cienciometria/index.i pynb

17 Es importante mencionar que, si bien es el método más eficiente, no permite alcanzar una cobertura del 100\% de la producción científica, puesto que no todos los artículos disponen de DOI. Sin embargo, la proporción de la literatura con el identificador es cada vez mayor: https://www.doi.org/factsheets/DOIKeyFacts.html

$18 \mathrm{El}$ oaDOI devuelve un enlace a una versión de OA legalmente disponible del artículo, cuando hay una disponible: http://un paywall.org/

19 Para la revisión del algoritmo utilizado se puede visitar el siguiente enlace: https://nbviewer.jupyter.org/github/restrepo/me dicion/blob/master/cienciometria/OpenAccess.ipynb

$20 \mathrm{El}$ algoritmo se encuentra disponible en https://colab.research.google.com/github/restrepo/medicion/blob/master/cienciom etria/OpenAccess.ipynb. La base de datos consolidada puede accederse en http://bit.ly/oadois-udea

21 Vía dorada: la definición de, si un artículo se considera de acceso cerrado o de acceso abierto, parte de las políticas de la revista. En el presente estudio esto se logró a partir del servicio Unpaywall (oaDOI.org).

$22 \mathrm{El} 4 \%$ restante corresponde a datos inconclusos frente al DOI. 
23 Ver: SciELO http://visual.scielo.org/v1/ y RedALyC https://www.redalyc.org/institucion.oa?id=15344\&tipo=coleccion

24 Aunque sea recomendación, como es el caso de la Universidad de Antioquia: http://roarmap.eprints.org/1946/

25 Siso-Calvo y Arquero-Avilés (2019, p.33) con respecto a la situación en España mencionan que:

En línea con las recomendaciones de la FECYT, es fundamental la búsqueda de fórmulas basadas en incentivos para publicar en abierto, de forma que se considere como mérito en los procesos de evaluación, así como el uso de indicadores de citación alternativos. En relación con el apoyo a los investigadores, es fundamental la formación para la adquisición de hábitos, competencias y actitudes para publicar en abierto, así como familiarizarse con las prácticas de propiedad intelectual y cesión de derechos de explotación. 